\title{
On an invariant distance induced by the Szegő Kernel
}

\author{
Steven G. Krantz ${ }^{1}$ Paweł M. Wójcicki² ${ }^{2}$
}

Received: 3 December 2020 / Accepted: 24 June 2021 / Published online: 13 July 2021

(c) The Author(s) 2021

\begin{abstract}
In this paper we introduce a new distance by means of the so-called Szegó kernel and examine some basic properties and its relationship with the so-called Skwarczyński distance. We also examine the relationship between this distance, and the so-called Bergman distance and Szegő distance.
\end{abstract}

Keywords Szegő kernel · Weighted Bergman kernel · Szegő distance · Bergman distance · Green’s function

Mathematics Subject Classification Primary 32A36 · Secondary 32A25

\section{Introduction}

In this paper, we introduce and describe some new distance by means of the Szegó kernel, called here by the Szegö projective distance and denoted by $\varrho_{\Omega}^{S}$. Since the Szegó kernel doesn't respect the transformation rule, we also consider the so-called Fefferman-Szegó kernel (described below) and the Fefferman-Szegö projective distance defined by it (denoted by $\left.S_{\Omega}^{S_{F}}\right)$. Both are defined on the same way and in a fashion similar to the so-called Skwarczyński distance (denoted by $\left.\varrho_{\Omega}\right)($ see $[1$, p. 20], and the definition actually based on ideas from projective geometry. The Skwarczyński distance is given more explicitly than the so-called Bergman distance and this is also our motivation too. Since this is new, we list and prove properties of this distance like completeness. The above considerations are nothing but natural generalizations of theorems valid in the case for the Bergman kernel and the Skwarczyński distance. We decided, however, to enclose it here for the sake of completeness.

Paweł M. Wójcicki

p.wojcicki@mini.pw.edu.pl

Steven G. Krantz

sk@math.wustl.edu

1 Department of Mathematics, Washington University in St. Louis, St. Louis, MO 63130, USA

2 Faculty of Mathematics and Information Science, Warsaw University of Technology, Koszykowa 75, 00-662 Warsaw, Poland
The new results can be found in Sect. 3.2.1 and at the end of the paper. The main results of the paper are Theorems 23 and 24 . We examine the relationship between completeness in the Szegó projective distance and completeness in the Skwarczyński distance.

\section{Definitions and notation}

Let $\Omega \subseteq \mathbb{C}^{n}$ be a bounded domain with $C^{2}$-smooth boundary. Let $A(\Omega)$ be those functions on $\bar{\Omega}$, which are both continuous on $\bar{\Omega}$ and holomorphic in $\Omega$. Denote by $H_{E}^{2}(\partial \Omega)$ the space consisting of the closure in the $L^{2}\left(\partial \Omega, d \sigma_{E}\right)$ topology of the restrictions to $\partial \Omega$ of elements of $A(\Omega)$ (here $d \sigma_{E}$ denotes the Euclidean surface area measure on $\partial \Omega$ ). Then $H_{E}^{2}(\partial \Omega)$ is a proper Hilbert subspace of $L^{2}\left(\partial \Omega, d \sigma_{E}\right)$. Recall that each element $f \in H_{E}^{2}(\partial \Omega)$ has a natural holomorphic extension to $\Omega$ given by its Poisson integral (see [2, p. 66]). The Szegó kernel $S(z, w)$ is the reproducing kernel for $H_{E}^{2}(\partial \Omega)$, that is

$f(z)=\int_{\partial \Omega} S(z, w) f(w) d \sigma_{E}(w), \quad \forall f \in H_{E}^{2}(\partial \Omega)$,

The problem is that the Euclidean surface measure does not transform nicely under biholomorphic mappings. We deal with this problem by instead using the so-called Fefferman surface area measure $\sigma_{F}$ (see [3]), which is given by:

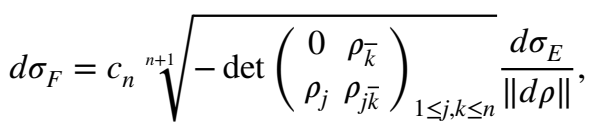


where $\rho_{j} \equiv \partial \rho / \partial z_{j}, \rho_{\bar{k}} \equiv \partial \rho / \partial \bar{z}_{k}, \rho_{j \bar{k}} \equiv \partial^{2} \rho / \partial z_{j} \partial \bar{z}_{k}$, and $\rho$ is a defining function for $\Omega$ (here $\|\cdot\|$ denotes the usual Euclidean distance). The constant $c_{n}$ is a dimensional constant (see [3]). We should consider the space $H_{F}^{2}(\partial \Omega)$ defined in the same way as $H_{E}^{2}(\partial \Omega)$ with $d \sigma_{F}$ instead of $d \sigma_{E}$. The space $H_{F}^{2}(\partial \Omega)$ is a Hilbert space with reproducing kernel in the sense of Aronszajn (see [4]). So it has the reproducing kernel $S_{F}(z, w)$. Of course, this new kernel is in general not the same as the usual Szegó kernel, but it certainly obeys the reproducing property (see [2, p. 66] and [5]):

$f(z)=\int_{\partial \Omega} S_{F}(z, w) f(w) d \sigma_{F}(w), \quad \forall f \in H_{F}^{2}(\partial \Omega)$.

Throughout the paper we are working with both $S_{F}$ and $S$. We always try to highlight what kernel is actually considered. When considering $S$ (denoted also by $S_{\Omega}$ ) we automatically assume that $\Omega$ is a bounded domain with $C^{2}$-smooth boundary in $\mathbb{C}^{n}$. If $S_{F}$ (denoted also by $S_{F, \Omega}$ ) is considered, $\Omega \Subset \mathbb{C}^{n}$ is assumed to be strongly pseudoconvex with $C^{\infty}$ -smooth boundary.

\section{The Fefferman-Projective Szegö distance and some remarks}

It turns out that, like the Bergman kernel, the Fefferman-Szegó kernel respects the so-called transformation rule (see ([5], Prop. 2 and also [6], Prop. 3.3):

Proposition 1 Let $\Omega_{1}, \Omega_{2} \subset \mathbb{C}^{n}$ and $\varphi: \Omega_{1} \rightarrow \Omega_{2}$ be a biholomorphic mapping. Assume there exists a well-defined holomorphic branch of $\left(\operatorname{det} J_{\mathbb{C}} \varphi(z)\right)^{n /(n+1)}$ on $\Omega_{1}$. Then we have

$$
\begin{aligned}
S_{F, \Omega_{1}}(z, w)= & S_{F, \Omega_{2}}(\varphi(z), \\
& \varphi(w))\left(\operatorname{det} J_{\mathbb{C}} \varphi(z)\right)^{n / n+1}\left(\overline{\operatorname{det} J_{\mathbb{C}} \varphi(w)}\right)^{n / n+1},
\end{aligned}
$$

where $S_{F, \Omega_{j}}(z, w)$ is the Fefferman-Szegö kernel on $\Omega_{j}$ for $j=1,2$.

This property leads us to the biholomorphically invariant distance induced by the Fefferman-Szegô kernel. We have to point out here that for $n>1$ the classical Szegó kernel doesn't obey the above transformation rule.

In order to introduce the distance, we recall some ideas from the theory of Hilbert spaces.

Let $(H,\langle\cdot, \cdot\rangle)$ be an arbitrary separable Hilbert space. Let us consider the following relation between two nonzero elements: $x \sim y$ if and only if there exists a complex constant $c \neq 0$ such that $x=c y$. The set of equivalence classes forms the (generally infinite dimensional) projective Hilbert space $P(H)$. This is a complete metric space with respect to the distance
$d_{H}([x],[y])=\operatorname{dist}\left([x] \cap S_{H},[y] \cap S_{H}\right)$,

where $S_{H} \subset H$ is the unit sphere, and as usual $\operatorname{dist}(A, B)=\inf \{d(x, y) \mid x \in A, y \in B\}$ for two nonempty subsets $A, B$ of $H$. Explicitly,

$$
\begin{aligned}
d_{H}^{2}([x],[y]) & =\inf _{\varphi, \phi \in[0,2 \pi]}\left\|\frac{e^{i \varphi} x}{\|x\|}-\frac{e^{i \phi} y}{\|y\|}\right\|^{2} \\
& =\inf _{\varphi, \phi \in[0,2 \pi]}\left[2-2 \operatorname{Re} \frac{e^{i(\varphi-\phi)}\langle x, y\rangle}{\|x\|\|y\|}\right] \\
& =2-2\left[\frac{\langle x, y\rangle\langle y, x\rangle}{\langle x, x\rangle\langle y, y\rangle}\right]^{1 / 2}
\end{aligned}
$$

where $\langle\cdot, \cdot\rangle$ denotes the scalar product of the Hilbert space $H$. Using this idea, M. Skwarczyński introduced in [1, p. 20] the biholomorphically invariant pseudodistance on domains in $\mathbb{C}^{n}$. It is directly based on the so-called Bergman kernel (see for example [7, p. 410] and [2, p. 49]). At first, we need an analogue of this idea for Szegô kernels.

Note that $S_{F}(z, z)$ does not vanish at any point $z \in \Omega$ (see [2, p. 66]). Define the map $\tau: \Omega \rightarrow P\left(H_{F}^{2}(\partial \Omega)\right.$ ) by the formula

$\tau(z):=\left[S_{F}(\cdot, z)\right]$.

This enables us to introduce the following continuous pseudodistance on $\Omega \times \Omega$ :

$$
\begin{aligned}
\varrho_{\Omega}^{S_{F}}(z, w) & :=\frac{1}{\sqrt{2}} d_{H^{2}(\partial \Omega)}(\tau(z), \tau(w)) \\
& =\left(1-\frac{\left|S_{F}(z, w)\right|}{\sqrt{S_{F}(z, z)} \sqrt{S_{F}(w, w)}}\right)^{1 / 2} .
\end{aligned}
$$

(we recall that the symbol $\varrho_{\Omega}$ is fixed for the so-called Skwarczyński distance (see [1, p. 20])).

Remark 2 Observe that the following conditions are equivalent:

(a) $\tau$ is injective;

(b) for each two distinct points $z, w \in \Omega$ the functions $S_{F}(\cdot, z), S_{F}(\cdot, w)$ are linearly independent;

(c) $\varrho_{\Omega}^{S_{F}}$ is a distance.

Let us note the following:

Remark 3 Since $\Omega$ is bounded, $\varrho_{\Omega}^{S_{F}}$ is a distance.

Proof of the Remark 3 Let $w, t \in \Omega, w \neq t$. The points $w$ and $t$ differ by at least one coordinate, let us say the $k$ th one. The polynomial $g(z)=z_{k}-w_{k}$ is an element of $H_{F}^{2}(\partial \Omega)$ and 
$g(w)=0, g(t) \neq 0$. Let us note now that point evaluations $E_{t}$ and $E_{w}$ are linearly independent. Indeed, if

$\lambda E_{t}(f)+\beta E_{w}(f)=0$

for all $f \in H_{F}^{2}(\partial \Omega)$, then for $f=g$ we have that $\lambda=0$. The choice $f \equiv 1$ implies that $\beta=0$, which shows that $E_{t}$ and $E_{w}$ are linearly independent. Since the transformation given in the Riesz Representation Theorem, which assigns to any linear, continuous functional its representing vector, is an antilinear isometry, then the vectors $S_{F, \Omega}(\cdot, t)$ and $S_{F, \Omega}(\cdot, w)$ are linearly independent. The conclusion follows now from Remark 2.

We call $\varrho_{\Omega}^{S_{F}}$ the Fefferman-Szegö projective distance (taking $K_{\Omega}$-the regular Bergman kernel instead of $S_{\Omega}$, we get the so-called Skwarczyński distance-see [1, p. 20]). The advantage of this distance is that, compared to the (regular) Szegő distance (given by the Szegó metricsee [5]), it is given in a more explicit way and thus seems to be advantageous from the computational point of view. Moreover, it is uniquely determined by the real analytic function

$H(z, w)=\frac{S_{F}(z, w) S_{F}(w, z)}{S_{F}(z, z) S_{F}(w, w)}$

on $\Omega \times \Omega$.

Remark 4 We define the Szegö projective distance on the same way just taking $S_{\Omega}$ instead of $S_{F, \Omega}$. We note, however, that in contrast to $\varrho_{\Omega}^{S_{F}}$, the distance $\rho_{\Omega}^{S}$ is not biholomorphically invariant. We call $\rho_{\Omega}^{S_{F}}$ the Fefferman-Szegö projective distance, and $\varrho_{\Omega}^{S}$ the Szegő projective distance.

Remark 5 We see that, for any biholomorphic mapping $\varphi: \Omega_{1} \rightarrow \Omega_{2}$, we have

$\varrho_{\Omega_{1}}^{S_{F}}(z, w)=\varrho_{\Omega_{2}}^{S_{F}}(\varphi(z), \varphi(w))$.

The proof follows from the transformation rule for the Szego kernel (see Proposition 1).

\subsection{The Fefferman-Szegő projective distance on the unit ball}

Let $\mathbb{B}^{n}=\left\{z \in \mathbb{C}^{n}: \rho(z):=|z|^{2}-1<0\right\} \subset \mathbb{C}^{n}$. Then the Szegó kernel for the unit ball $\mathbb{B}^{n}$ is given by

$S_{F}(z, w)=\frac{(n-1) !}{2 \pi^{n}} \frac{1}{(1-z \cdot \bar{w})^{n}}$.
From the formula (1) it follows directly that

$$
\left(\varrho_{\mathbb{B}^{n}}^{S_{F}}\right)^{2}(z, w)=1-\left(\frac{\left(1-|z|^{2}\right)\left(1-|w|^{2}\right)}{|1-z \cdot \bar{w}|^{2}}\right)^{n / 2} .
$$

$\varrho_{\mathbb{B}^{2}}^{S_{F}}(z, w)=\frac{|z-w|^{2}}{|1-z \cdot \bar{w}|^{2}}$

Recall now that the Skwarczyński distance for the unit disc in $\mathbb{C}$ is

$\varrho_{\mathbb{D}}(z, w)=\left|\frac{z-w}{1-z \bar{w}}\right|$

(see [1, p. 21]). Thus

$\varrho_{\mathbb{B}^{2}}^{S_{F}}\left(\left(z_{1}, 0\right),\left(w_{1}, 0\right)\right)=\varrho_{\mathbb{D}}^{2}\left(z_{1}, w_{1}\right)$.

Moreover, for $n=1$, we have

$\varrho_{\mathbb{D}}(z, w)=\varrho_{\mathbb{B}^{1}}^{S_{F}}(z, w) \sqrt{2-\left(\varrho_{\mathbb{B}^{1}}^{S_{F}}\right)^{2}(z, w)}$.

Remark 6 The same formulas hold for $\varrho^{S}$.

\subsection{Completeness with respect to the $Q_{\Omega}^{S}$ distance}

In this subsection we are interested in the Szegő projective distance rather than Fefferman-Szegó projective distance. The reason is that arguments from this subsection repeated for the Fefferman-Szegó projective distance give that every strongly pseudoconvex domain with smooth bound-

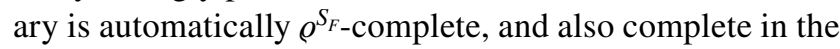
Szegő metric (introduced in [5]).

We list here some important theorems which are directly taken from the Bergman kernel theory (see [7, Theorem 12.9.6.] for instance). We are doing this for the sake of completeness of the paper.

Following ideas of [8] and particularly [1, p. 22] we can study completeness with respect to the invariant distance. Additionally, we will prove now that the so-called Kobayashi condition implies $\varrho_{\Omega}^{S}$-completeness.

Theorem 7 A sequence $\left(z_{m}\right) \in \Omega, m=1,2, \ldots$, is Cauchy with respect to the distance $\varrho_{\Omega}^{S}$ if and only if the sequence $\tau\left(z_{m}\right)$ is Cauchy in $P\left(H^{2}(\partial \Omega)\right)$.

Proof This is a direct consequence of the definition of $\varrho_{\Omega}^{S}$.

Theorem 8 A sequence $z_{m} \in \Omega, m=1,2, \ldots$, is Cauchy with respect to $\varrho_{\Omega}^{S}$ if and only if there exists an $f \in H^{2}(\partial \Omega)$ such that $\|f\|_{H^{2}}=1$ and 
$\lim _{m \rightarrow \infty} \frac{\left|f\left(z_{m}\right)\right|^{2}}{S_{\Omega}\left(z_{m}, z_{m}\right)}=1$.

Proof By the previous theorem, a sequence $\left(z_{m}\right)$ is Cauchy in $\Omega$ if and only if $\left(\tau\left(z_{m}\right)\right)$ is Cauchy in $P\left(H^{2}(\partial \Omega)\right)$. By completeness of $P\left(H^{2}(\partial \Omega)\right)$, the sequence $\left(\tau\left(z_{m}\right)\right)$ converges to some $[f]$. We may assume that $\|f\|_{H^{2}}=1$. Thus

$\lim _{m \rightarrow \infty} d_{H^{2}(\partial \Omega)}\left(\tau\left(z_{m}\right),[f]\right)=0$

but this is equivalent (by the definition) to (2). The reverse implication is a direct consequence of the definition of $\varrho_{\Omega}^{S}$.

Theorem 9 (see p. 494 in [7]) The Euclidean distance and $\varrho_{\Omega}^{S}$ induce the same topology in $\Omega$.

Proof Assume that $z_{j} \in \Omega$ converges to $z \in \Omega$ in the Euclidean norm. Then $\lim _{j \rightarrow \infty} \varrho_{\Omega}^{S}\left(z_{j}, z\right)=0$ since the Szegő function is continuous. Conversely, $\lim _{j \rightarrow \infty} \varrho_{\Omega}^{S}\left(z_{j}, z\right)=0$ implies that

$\lim _{j \rightarrow \infty}\left\|\frac{e^{i \theta_{j}} S_{\Omega}\left(\cdot, z_{j}\right)}{\sqrt{S_{\Omega}\left(z_{j}, z_{j}\right)}}-\frac{S_{\Omega}(\cdot, z)}{\sqrt{S_{\Omega}(z, z)}}\right\|_{H^{2}}=0$,

where $\left(\theta_{j}\right)$ is a suitable sequence of real numbers. Thus there exist constants $c_{j} \neq 0, j=1,2, \ldots$, such that $c_{j} S_{\Omega}\left(\cdot, z_{j}\right) \stackrel{H^{2}}{\longrightarrow} S_{\Omega}(\cdot, z)$. Since $1 \in H^{2}(\partial \Omega)$, we see that

$\lim _{j \rightarrow \infty} \bar{c}_{j}=\lim _{j \rightarrow \infty}\left(1, c_{j} S_{\Omega}\left(\cdot, z_{j}\right)\right)_{\mu}=\left(1, S_{\Omega}(\cdot, z)\right)_{H^{2}}=1$.

Let $\pi_{k}$ denote the $k$ th coordinate function. We have

$$
\begin{aligned}
& \lim _{j \rightarrow \infty} \pi_{k}\left(z_{j}\right) \\
& \quad=\lim _{j \rightarrow \infty}\left(\pi_{k}(\cdot), S_{\Omega}\left(\cdot, z_{j}\right)\right)_{\mu}=\lim _{j \rightarrow \infty} \frac{1}{\bar{c}_{j}}\left(\pi_{k}(\cdot), c_{j} S_{\Omega}\left(\cdot, z_{j}\right)\right)_{H^{2}} \\
& \quad=\left(\pi_{k}(\cdot), S_{\Omega}(\cdot, z)\right)_{H^{2}}=\pi_{k}(z), \quad \text { i.e. } \quad \lim _{j \rightarrow \infty} z_{j}=z .
\end{aligned}
$$

Hence the two topologies coincide. Having this result in hand, we can prove (in a fashion similar to that for the Bergman kernels see $\left[9\right.$, p. 93]) that the $\rho_{\Omega}^{S}$ completeness is closely related to the dimension of $H^{2}(\partial \Omega)\left(L_{H}^{2}(\partial \Omega)\right)$.

Theorem 10 If $\Omega$ is $\varrho_{\Omega}^{S}$ complete, then $\operatorname{dim} H^{2}(\partial \Omega)=\infty$.

Proof We can adapt the proof in [9, p. 93]. Assume that $\operatorname{dim} H^{2}(\partial \Omega)<\infty$. Then the closed unit ball in $H^{2}(\partial \Omega)$ is compact. Let $g_{z}(\cdot)=\frac{S_{\Omega}(\cdot, z)}{\sqrt{S_{\Omega}(z, z)}}$, where $z \in \Omega$. Then

$$
\begin{aligned}
\left\|g_{z}\right\|^{2} & =\int_{\Omega} g_{z}(w) \overline{g_{z}(w)} \mu(w) \mathrm{d} V \\
& =\int_{\Omega} \frac{S_{\Omega}(w, z)}{\sqrt{S_{\Omega}(z, z)}} \frac{\overline{S_{\Omega}(w, z)}}{\sqrt{S_{\Omega}(z, z)}} \mathrm{d} V \\
& =\frac{1}{S_{\Omega}(z, z)} \int_{\Omega} S_{\Omega}(w, z) \overline{S_{\Omega}(w, z)} \mathrm{d} V \\
& =\frac{1}{S_{\Omega}(z, z)} S_{\Omega}(z, z) . \\
& =1
\end{aligned}
$$

If $\left(z_{k}\right)_{k=1}^{\infty} \rightarrow z_{0} \in \partial \Omega$ (in the usual Euclidean topology), then (by compactness of the unit ball) $\left(g_{z_{k j}}\right)_{j=1}^{\infty}$ has a subsequence that is convergent to $g \in H^{2}(\partial \Omega)$, where $\|g\|_{H^{2}}=1$. Denote this sequence by $\left(g_{z_{k}}\right)_{k=1}^{\infty}$. Let us see that $\left(z_{k}\right)_{k=1}^{\infty}$ is $\varrho_{\Omega}^{S}$-Cauchy. Indeed

$$
\begin{aligned}
& \left|\left\langle g_{z_{m}}, g_{z_{n}}\right\rangle\right| \\
& =\left|\int_{\Omega} g_{z_{m}}(w) \overline{g_{z_{n}}(w)} \mu(w) \mathrm{d} V\right| \\
& =\left|\int_{\Omega} \frac{S_{\Omega}\left(w, z_{m}\right)}{\sqrt{S_{\Omega, \mu}\left(z_{m}, z_{m}\right)}} \frac{S_{\Omega}\left(w, z_{n}\right)}{\sqrt{S_{\Omega}\left(z_{n}, z_{n}\right)}} \mu(w) \mathrm{d} V\right| \\
& =\frac{1}{\sqrt{S_{\Omega}\left(z_{m}, z_{m}\right)} \sqrt{S_{\Omega, \mu}\left(z_{n}, z_{n}\right)}}\left|\int_{\Omega} S_{\Omega}\left(w, z_{m}\right) \overline{S_{\Omega}\left(w, z_{n}\right)} \mu(w) \mathrm{d} V\right| \\
& =\frac{\left|S_{\Omega}\left(z_{m}, z_{n}\right)\right|}{\sqrt{S_{\Omega}\left(z_{m}, z_{m}\right)} \sqrt{S_{\Omega}\left(z_{n}, z_{n}\right)}},
\end{aligned}
$$

i.e.

$\left(\varrho_{\Omega}^{S}\right)^{2}\left(z_{m}, z_{n}\right)=1-\left|\left\langle g_{z_{m}}, g_{z_{n}}\right\rangle\right|$

Since the term on the right hand side tends to 0 when $m, n \rightarrow \infty$ we conclude that $\varrho_{\Omega}^{S}\left(z_{m}, z_{n}\right)<\epsilon$ for $m, n$ large enough. Thus we found a $\varrho_{\Omega}^{S}$-sequence which has the limit $z_{0} \in \partial \Omega$. This should not happen since $\Omega$ is assumed to be $\varrho_{\Omega}^{S}$-complete.

Some of the ideas below-particularly Theorems 11 and 12-follow upon the ones in [1, p. 23, 24] .

Theorem 11 (Szegő version of the Kobayashi theorem) Assume that, for every sequence $\left(z_{m}\right) \in \Omega$ without an accumulation point in $\Omega$ and for every $f \in H^{2}(\partial \Omega)$,

$\lim _{m \rightarrow \infty} \frac{\left|f\left(z_{m}\right)\right|^{2}}{S_{\Omega}\left(z_{m}, z_{m}\right)}=0$.

Then $\Omega$ is $\varrho_{\Omega}^{S}$-complete.

Proof Suppose that $\left(z_{m}\right) \in \Omega$ is a Cauchy sequence without limit in $\Omega$. Thus $\left(z_{m}\right)$ has no accumulation point in $\Omega$, and 
(3) holds. But (3) contradicts (2). Thus there is a limit point of $\left(z_{m}\right)$ in $\Omega$.

The hypothesis of the above theorem applied to the Bergman kernel $K$ instead of $S$, and to the Szegô space $H^{2}(\partial \Omega)$ instead of $L_{H}^{2}(\partial \Omega)$, is the so-called Kobayashi condition (see [8]). Kobayashi showed that this condition implies that the considered domain is Bergman complete. Skwarczyński has a proof that this condition implies $\varrho_{\Omega}$-completeness- - [10] (see Sect. 3.4 for the definition of $\varrho_{\Omega}$ ).

Theorem 12 Suppose that, for each boundary point $z_{0} \in \partial \Omega$ (of a bounded domain $\Omega$ with $C^{2}$-smooth boundary), there is a function $h \in \mathcal{O}(\Omega)$ such that

(a) $|h(z)|<1$ for $z \in \Omega$,

(b) $\lim _{z \rightarrow z_{0}}|h(z)|=1$.

Then $\Omega$ is complete with respect to $\varrho_{\Omega}^{S}$.

Proof Let $\left(z_{m}\right)$ be a sequence with no accumulation point in $\Omega$. It suffices to show that, for any $f \in H^{2}(\partial \Omega)$,

$\lim _{m \rightarrow \infty} \frac{\left|f\left(z_{m}\right)\right|^{2}}{S_{\Omega}\left(z_{m}, z_{m}\right)}=0$.

We may assume that $z_{m} \rightarrow z \in \partial \Omega$. For any $\epsilon>0$, there is $k$ such that $\left\|h^{k} f\right\|_{H^{2}}^{2}<\epsilon$ (by the Lebesgue dominated convergence theorem). If $m$ is large enough, then

$(1-\epsilon)\left|f\left(z_{m}\right)\right|^{2} \leq\left|h^{k}\left(z_{m}\right) f\left(z_{m}\right)\right|^{2} \leq S_{\Omega}\left(z_{m}, z_{m}\right)|| h^{k} f \|_{H^{2}}^{2}$.

Thus

$\frac{\left|f\left(z_{m}\right)\right|^{2}}{S_{\Omega}\left(z_{m}, z_{m}\right)} \leq \frac{\epsilon}{1-\epsilon}$.

Recall the definition of a peak point with respect to (some family) $\mathcal{F}$.

Definition 13 Let $D$ be a bounded domain in $\mathbb{C}^{n}$. A boundary point $z_{0} \in \partial D$ is called a peak point with respect to $\mathcal{F} \subset C(\bar{D})$ if there is $h \in \mathcal{F}$ such that
(a) $h\left(z_{0}\right)=1$
(b) $|h(z)|<1$ on $\bar{D} \backslash\left\{z_{0}\right\}$

Recall some classical results concerning peak points.

Theorem 14 (cf. [7, p. 802]; [11, 12]) If D is a strongly pseudoconvex domain in $\mathbb{C}^{n}$ and $z_{0} \in \partial D$, then $z_{0}$ is a peak point with respect to $\mathcal{O}(\bar{D})$. If $D$ is a strongly pseudoconvex domain in $\mathbb{C}^{n}$ with a smooth boundary and $z_{0} \in \partial D$ then $z_{0}$ is a peak point with respect to $\mathcal{O}(D) \cap C(\bar{D})$. Moreover, if $D$ is a bounded pseudoconvex domain in $\mathbb{C}^{2}$ with real analytic boundary, then any boundary point $z_{0} \in \partial D$ is a peak point with respect to $\mathcal{O}(D) \cap C(\bar{D})$.

We infer from this and Theorem 12 the following:

Corollary 15 Every strongly pseudoconvex domain $\Omega$ in $\mathbb{C}^{n}$ with $C^{2}$-smooth boundary is complete with respect to $\rho_{\Omega}^{S}$. Moreover, every bounded pseudoconvex domain $\Omega$ in $\mathbb{C}^{2}$ with real analytic boundary is complete with respect to $\rho_{\Omega}^{S}$.

Remark 16 So now one can clearly note, that the above arguments, repeated for $S_{F}$ rather than $S$ provide every strongly pseudoconvex domain with $C^{\infty}$-smooth boundary is automatically complete in the $\rho_{\Omega}^{S_{F}}$ distance.

\subsubsection{Comparison of the Bergman and Szegő kernels off the diagonal}

Using recent estimates obtained in [13] we may estimate the quotient $\left|S_{\Omega}(z, w) / K_{\Omega}(z, w)\right|$ on domains which are not $\varrho_{\Omega}$ -complete (Skwarczyński distance). Note that, if $\left(z_{n}\right)_{n=1}^{\infty}$ is a $\varrho_{\Omega^{-}}$Cauchy sequence then, for any $\epsilon>0$,

$1-\epsilon \leq \frac{\left|K_{\Omega}\left(z_{n}, z_{m}\right)\right|}{\sqrt{K_{\Omega}\left(z_{n}, z_{n}\right)} \sqrt{K_{\Omega}\left(z_{m}, z_{m}\right)}} \leq 1$

if only $m, n$ are large enough. We now have the following:

Theorem 17 If $\Omega \Subset \mathbb{C}^{n}$ is a pseudoconvex domain with $C^{2}$ -smooth boundary and which is not $\varrho_{\Omega}$-complete then

$\lim _{n \rightarrow \infty}\left|\frac{S\left(z_{n}, z_{m}\right)}{K\left(z_{n}, z_{m}\right)}\right|=0$

for any $\varrho_{\Omega^{-}}$Cauchy sequence $\left(z_{p}\right)_{p=1}^{\infty}$, where $m$ is large enough.

Proof Let $\epsilon>0$. Then for $n, m$ large enough we have

$\left|\frac{K_{\Omega}\left(z_{n}, z_{m}\right)}{S_{\Omega}\left(z_{n}, z_{m}\right)}\right|$

$=\frac{\left|K_{\Omega}\left(z_{n}, z_{m}\right)\right|}{\sqrt{K_{\Omega}\left(z_{n}, z_{n}\right)} \sqrt{K_{\Omega}\left(z_{m}, z_{m}\right)}} \frac{\sqrt{K_{\Omega}\left(z_{n}, z_{n}\right)} \sqrt{K_{\Omega}\left(z_{m}, z_{m}\right)}}{\sqrt{S_{\Omega}\left(z_{n}, z_{n}\right)} \sqrt{S_{\Omega}\left(z_{m}, z_{m}\right)}}$.

$\frac{\sqrt{S_{\Omega}\left(z_{n}, z_{n}\right)} \sqrt{S_{\Omega}\left(z_{m}, z_{m}\right)}}{\left|S_{\Omega}\left(z_{m}, z_{n}\right)\right|} \underset{n \rightarrow \infty}{\longrightarrow} \infty$ 
(since $\frac{S(z, z)}{K(z, z)} \leq c \delta(z)|\ln (\delta(z))|^{\alpha} \underset{z \rightarrow \partial \Omega}{\longrightarrow} 0$-see [13]).

Thus we have

Corollary 18 If $\Omega \Subset \mathbb{C}^{n}$ is a pseudoconvex domain with $C^{2}$ -smooth boundary such that its Bergman kernel $K_{\Omega}(z, w)$ satisfies $\left|K_{\Omega}(z, w)\right| \leq M,\left|S_{\Omega}(z, w)\right|>0$ on $\bar{\Omega} \times \bar{\Omega} \backslash F$, where $F=\{(z, w) \in \partial \Omega \times \partial \Omega, z=w\}$ then $\Omega$ is $\varrho_{\Omega^{-}}$complete, and thus Bergman complete.

In particular, every strongly pseudoconvex domain $\Omega \Subset \mathbb{C}^{n}$ with $C^{\infty}$-boundary is $\varrho_{\Omega}$-complete (see [14]) (we know this already from Corollary 15 applied to the Bergman kernel—see [1, p. 25]).

\subsection{Relation of $Q^{S}$ to the Skwarczyński distance}

It turns out that $\varrho_{\Omega}^{S}$ is related to $\varrho_{\Omega}$ (Skwarczyński distance) by some biholomorphic invariants introduced below. Let us recall that $\rho_{\Omega}^{S}$, by its definition, is uniquely determined by the real analytic function

$H_{\Omega}(z, w)=\frac{S_{\Omega}(z, w) S_{\Omega}(w, z)}{S_{\Omega}(z, z) S_{\Omega}(w, w)}$.

Define $L_{\Omega}(z, w)$ a corresponding quotient for the Bergman kernel, i.e.

$L_{\Omega}(z, w)=\frac{K_{\Omega}(z, w) K_{\Omega}(w, z)}{K_{\Omega}(z, z) K_{\Omega}(w, w)}$.

Now define a new biholomorphically invariant $H L_{\Omega}(z, w)$ by

$H L_{\Omega}(z, w)=\frac{L_{\Omega}(z, w)^{n}}{H_{\Omega}(z, w)^{n+1}}=\frac{\left|K_{\Omega}(z, w)\right|^{2 n}}{\left|S_{\Omega}(z, w)\right|^{2 n+2}} \frac{S_{\Omega}(z, z)^{n+1}}{K_{\Omega}(z, z)^{n}} \frac{S_{\Omega}(w, w)^{n+1}}{K_{\Omega}(w, w)^{n}}$

We can write it by means of another biholomorphic invariant $S K_{\Omega}(z, w)$, where

$S K_{\Omega}(z, w)=\frac{S_{\Omega}(z, w)^{n+1}}{K_{\Omega}(z, w)^{n}} \quad z, w \in \Omega$

introduced (for $S_{F, \Omega}$ in fact) in [5, formula (3.1)]. See also [13]. Now

$H L_{\Omega}(z, w)=\frac{1}{\left|S K_{\Omega}(z, w)\right|^{2}} S K_{\Omega}(z, z) S K_{\Omega}(w, w)$.

By its definition, $H L_{\Omega}$ is a symmetric, real analytic function on $\Omega \times \Omega$. Moreover
Lemma 19 The following holds:

(a) For any $z \in \Omega, H L_{\Omega}(z, z)=1$

(b) $H L_{\Omega}(z, w)=\frac{\left(1-\rho^{2}(z, w)\right)^{2 n}}{\left(1-\left(\rho^{S}\right)^{2}(z, w)\right)^{2 n+2}}$

(c) $H L_{\mathbb{B}^{n}}(z, w)=1$ for all $z, w \in \mathbb{B}^{n}$.

Proof Properties $(a),(b),(c)$ follows directly from the definition of $H L$ and from the formulas:

$$
\begin{aligned}
S_{\Omega}(z, w) & =\frac{(n-1) !}{2 \pi^{n}} \frac{1}{(1-z \cdot \bar{w})^{n}} \quad, \quad K_{\Omega}(z, w) \\
& =\frac{1}{\operatorname{vol}(\mathbb{B})} \frac{1}{(1-z \cdot \bar{w})^{n+1}} .
\end{aligned}
$$

However, the Szegó kernel $S_{\Omega}$ itself blows up on the boundary. Indeed :

Remark 20 Similarly to the Bergman kernel $K_{\Omega}$, the Szegó kernel $S_{\Omega}$ satisfies

$\lim _{z \rightarrow \partial \Omega} S_{\Omega}(z, z)=\infty$

Proof By the definition (see [5]),

$S K_{\Omega}(z, z)=\frac{S(z, z)^{n+1}}{K(z, z)^{n}}=S(z, z) \frac{S(z, z)^{n}}{K(z, z)^{n}}$

But $\frac{S_{\Omega}(z, z)}{K_{\Omega}(z, z)} \leq c \delta(z)|\ln (\delta(z))|^{\alpha} \underset{z \rightarrow \partial \Omega}{\longrightarrow} 0$-see [13], and (as previously) $\lim _{z \rightarrow \partial \Omega} S K_{\Omega}(z, z)=$ constant $>0$.

One can note that both $\varrho_{\Omega}(z, w)$ and $\varrho_{\Omega}^{S}(z, w)$ tend to 1 for $w \rightarrow \partial \Omega, z \neq w$. But, in view of Lemma 19 , the quantity $\rho$ has stronger boundary asymptotic properties than $\rho^{S}$.

Remark 21 When considering $\{\Omega=\rho<0\} \Subset \mathbb{C}^{n}$ a strongly pseudoconvex domain with $C^{\infty}$ boundary, and $S_{F, \Omega}$ rather than $S_{\Omega}$ beside of the above properties one also has that

Proposition 22 If $S_{F, \Omega}(z, w) \neq 0$ for $z \in \Omega$ and any $w \in \partial \Omega$ then $\lim _{w \rightarrow \partial \Omega} H L_{\Omega}(z, w)$ exists and is finite.

Proof Note first that since $\Omega$ is smoothly bounded, strongly pseudoconvex domain the assumptions are always fulfilled for $w$ in or near the boundary $\partial \Omega$ and $z$ near to $w$. This is because $S_{F, \Omega}(z, w)$ (as well as $K_{\Omega}(z, w)$ ) does not vanish in this case (as follows from [15]). Recall (see [5]) that, for a strongly pseudoconvex domain $\Omega$ with the defining function suitably normalized, 
$S_{F} K_{\Omega}(z, z)= \begin{cases}(n-1) ! /\left(c_{n}^{n+1}(n \pi)^{n}\right)+(n-3) ! q_{\Omega} r^{2} /\left(c_{n}^{n+1} n^{n}\right)+O\left(r^{3}\right), & n \geq 4 \\ 2 /\left(c_{3}^{4} 27 \pi^{3}\right)+q_{\Omega} r^{2} /\left(9 c_{3}^{4}\right)+O\left(r^{3}|\ln r|\right), & n=3 \\ 1 /\left(c_{2}^{3} 4 \pi^{2}\right)+\mu_{2} r^{2}+\mu_{3} r^{4} \ln r+3 \pi^{2} \tilde{q}_{\Omega}^{2} r^{6} \ln ^{2} r /\left(16 c_{2}^{3}\right)+O\left(r^{6}|\ln r|\right), & n=2\end{cases}$

for $z$ close to the boundary, where $\mu_{2}, \mu_{3} \in C^{\infty}(\bar{\Omega})$ and $q_{\Omega}, \tilde{q}_{\Omega}$ are certain local geometric boundary invariants and $r:=-\rho$. From the above follows

$\lim _{z \rightarrow \partial \Omega} S_{F} K_{\Omega}(z, z)=$ constant $>0$

Now, since the Szegó kernel $S_{F}$ (like the Bergman kernel $K$ ) extends continuously outside diagonal on $\partial \Omega \times \partial \Omega$ then, of course, $\left|S_{F, \Omega}(z, w)\right|>M>0,\left|K_{\Omega}(z, w)\right|<N$ (by the assumption from $(c)$ ) and so

$\frac{1}{\left|S_{F} K_{\Omega}(z, w)\right|^{2}}=\frac{\left|K_{\Omega}(z, w)\right|^{2 n}}{\left|S_{F, \Omega}(z, w)\right|^{2 n+2}} \leq N M<\infty$.

Putting this together and using the definition of $H L_{\Omega}$ we ends the proof.

Theorem 23 Assume that $\Omega$ is a $\rho_{\Omega}^{S}$-complete domain on which $H L_{\Omega}(z, w) \leq 1$ for all $z, w \in \Omega$. Then $\Omega$ is $\varrho_{\Omega}$-complete (that is, complete in the Skwarczyński distance).

Proof Let $\left(z_{n}\right)$ be any $\varrho$-Cauchy sequence. Then, for any $\epsilon_{1}>0$, we have that $\rho\left(z_{k}, z_{p}\right)<\epsilon_{1}$ if $k, p$ large enough. In view of Lemma 19b we have

$\epsilon_{1}^{2}>\rho_{\Omega}^{2}\left(z_{k}, z_{p}\right)=1-\sqrt[2 n]{H L\left(z_{k}, z_{p}\right)}\left(1-\left(\rho_{\Omega}^{S}\right)^{2}\left(z_{k}, z_{p}\right)\right)^{\frac{2 n+2}{2 n}}$,

which is equivalent to

$$
\frac{\left(1-\epsilon_{1}^{2}\right)^{\frac{2 n}{2 n+2}}}{\sqrt[2 n+2]{H L_{\Omega}\left(z_{k}, z_{p}\right)}} \leq 1-\left(\varrho_{\Omega}^{S}\right)^{2}\left(z_{k}, z_{p}\right)
$$

or

$\left(\rho_{\Omega}^{S}\right)^{2}\left(z_{k}, z_{p}\right)<1-\frac{\left(1-\epsilon_{1}^{2}\right)^{\frac{2 n}{2 n+2}}}{\sqrt[2 n+2]{H L_{\Omega}\left(z_{k}, z_{p}\right)}}$.

We want to show that $\left(z_{n}\right)$ is convergent by proving it is $\varrho_{\Omega}^{S}$-Cauchy. So, for any $\epsilon>0$, we want to have $\varrho_{\Omega}^{S}\left(z_{k}, z_{p}\right)<\epsilon$ for $k, p$ large enough. Note that $(\star)$ implies $\varrho_{\Omega}^{S}\left(z_{k}, z_{p}\right) \leq 1$, thus for $\epsilon \geq 1$ one has $\varrho_{\Omega}^{S}\left(z_{k}, z_{p}\right)<\epsilon$. For $\epsilon \in(0,1)$ one can pick any $\epsilon_{1}$ in $\left(0, \sqrt{1-\left(1-\epsilon^{2}\right)^{\frac{2 n+2}{2 n}} \sqrt[2 n]{H L_{\Omega}\left(z_{k}, z_{p}\right)}}\right)$. Note that the quantity under the square root is nonnegative, since
$H L_{\Omega}\left(z_{k}, z_{p}\right)<\frac{1}{\left(1-\epsilon^{2}\right)^{2 n+2}}$,

as we assumed that $H L_{\Omega} \leq 1$ on $\Omega \times \Omega$. Now, after squaring and rearranging, one has

$\left(1-\epsilon^{2}\right) \sqrt[2 n+2]{H L_{\Omega}\left(z_{k}, z_{p}\right)}<\left(1-\epsilon_{1}^{2}\right)^{\frac{2 n}{2 n+2}}$,

which implies by $(\star)$ that $\varrho_{\Omega}^{S}\left(z_{k}, z_{p}\right)<\epsilon$, for $k, p$ sufficiently large.

Theorem 24 Assume $\Omega$ is a $\varrho_{\Omega^{-}}$complete domain on which $H L_{\Omega}(z, w) \geq 1$ for all $z, w \in \Omega$. Then $\Omega$ is $\varrho_{\Omega}^{S}$-complete.

Proof Let $\left(z_{n}\right)$ be any $\rho^{S}$-Cauchy sequence. Then, for any $\epsilon_{1}>0$, we have that $\varrho_{\Omega}^{S}\left(z_{k}, z_{p}\right)<\epsilon_{1}$ if $k, p$ large enough. In view of Lemma $19 \mathrm{~b}$ we have

$\epsilon_{1}^{2}>\left(\varrho_{\Omega}^{S}\right)^{2}\left(z_{k}, z_{p}\right)=1-\frac{\left(1-\varrho_{\Omega}^{2}\left(z_{k}, z_{p}\right)\right)^{\frac{2 n}{2 n+2}}}{\sqrt[2 n+2]{H L_{\Omega}\left(z_{k}, z_{p}\right)}}$

which is equivalent to

$\left(1-\rho_{\Omega}^{2}\left(z_{k}, z_{p}\right)\right)^{\frac{2 n}{2 n+2}}>\left(1-\epsilon_{1}^{2}\right) \sqrt[2 n+2]{H L_{\Omega}\left(z_{k}, z_{p}\right)}$

or

$\varrho_{\Omega}^{2}\left(z_{k}, z_{p}\right)<1-\left(1-\epsilon_{1}^{2}\right) \sqrt[2 n+2]{\frac{2 n}{2 n}} \sqrt{H L_{\Omega}\left(z_{k}, z_{p}\right)}$.

We want to show that $\left(z_{n}\right)$ is convergent by proving it is $\rho_{\Omega}$ -complete. So, for any $\epsilon>0$, we want to have $\rho_{\Omega}\left(z_{k}, z_{p}\right)<\epsilon$ for $k, p$ large enough. Note that $(\star \star)$ implies that $\varrho_{\Omega}\left(z_{k}, z_{p}\right) \leq 1$, thus for $\epsilon \geq 1$ one has $\varrho_{\Omega}\left(z_{k}, z_{p}\right)<\epsilon$. For $\epsilon \in(0,1)$ one can pick any $\epsilon_{1}$ in $\left(0, \sqrt{1-\left(1-\epsilon^{2}\right)^{\frac{2 n}{2 n+2}}}\right)$. Note that the quantity under the square root is nonnegative, since $\epsilon>0$. That means

$\frac{1-\epsilon^{2}}{\left(1-\epsilon_{1}^{2}\right)^{\frac{2 n+2}{2 n}}}<1 \leq \sqrt[2 n]{H L_{\Omega}\left(z_{k}, z_{p}\right)}$,

which together with $(\star \star)$ yields $\varrho_{\Omega}\left(z_{k}, z_{p}\right)<\epsilon$.

Corollary 25 If $\Omega$ is a domain for which $H L_{\Omega}(z, w)=1$ for all $z, w \in \Omega$, then $\Omega$ is $\varrho$-complete if and only if $\Omega$ is $\rho_{\Omega}^{S}$ -complete. 
Thus we have derived a characterization of those domains on which $\varrho$-completeness is equivalent to $\varrho^{S}$ -completeness.

Corollary 26 For a domain $\Omega$ with $H L_{\Omega} \equiv 1$ on $\Omega \times \Omega$, consider the statements:

(1) $\Omega$ is o-complete.

(2) $\Omega$ is $O_{\Omega}^{S}$-complete.

(3) $\Omega$ is Bergman complete.

Then $(1) \Longleftrightarrow(2),(2) \Rightarrow(3)$.

The proof of $(1) \Rightarrow(3)$ is given in [16]. According to our knowledge it is still open question whether (3) $\Rightarrow(1)$.

\subsection{Relation of the Fefferman-Szegö projective distance $Q^{S_{F}}$ to the Bergman distance and the Szegö distance}

Assume that now we do consider the Fefferman-Szegó kernel $S_{F}$. Let us recall that the Bergman metric $F_{B}$ on $\Omega$ at $z$ in the direction vector $\xi$ based at $z, F_{B}(z, \xi)$ is related to the Fefferman-Szegó metric $F_{S_{F}}(z, \xi)$ by

$m_{\Omega} F_{S_{F}}(z, \xi) \leq F_{B}(z, \xi)<M_{\Omega} F_{S_{F}}(z, \xi)$

where $0<m_{\Omega}<M_{\Omega}<\infty$ and $z \in \Omega$ and $\xi \in T \Omega$ (see [5, Theorems 3-5], [15, 17]).

Denote by $s_{F}(z, w)$ and $b(z, w)$ the distances induced by the Szegó and Bergman metric respectively (on the standard way—see [7] (p.482) for instance).

Theorem 27 There are some positive constants $c, \widetilde{m(\Omega)}, \widetilde{M(\Omega)}$, such that for every $z, w \in \Omega$ one has:

$\varrho_{\Omega}^{S_{F}}(z, w) \leq c \cdot s_{F}(z, w) \leq \widetilde{m(\Omega)} b(z, w) \leq \widetilde{M(\Omega)} s_{F}(z, w)$.

Proof This clearly follows from the estimation (2) and techniques analogous to the ones used for the Bergman kernel in [16].

\section{The relationship between $H L$, the Bergman metric and the Fefferman- Szegő metric}

In this section we get an exact connection between the Bergman and Szegó metrics by means of the quantity given by $H L_{\Omega}$. The key idea is a simple remark. Note that, by the definition, we have
$L_{\Omega}(z, w)=\left(1-\varrho_{\Omega}^{2}(z, w)\right)^{2}$

or just

$\frac{\left|K_{\Omega}(z, w)\right|^{2}}{\left(1-\rho_{\Omega}^{2}(z, w)\right)^{2}}=K_{\Omega}(z, z) K_{\Omega}(w, w)$,

for $z, w \in \Omega$. Taking the natural logarithm $\ln$ on both sides, one gets

$$
\begin{aligned}
& \ln K_{\Omega}(z, z)+\ln K_{\Omega}(w, w)=\ln K_{\Omega}(z, w)+\ln K_{\Omega}(w, z) \\
& \quad-2 \ln \left(1-\rho_{\Omega}^{2}(z, w)\right),
\end{aligned}
$$

so

$\frac{\partial^{2}}{\partial z_{i} \partial \bar{z}_{j}} \ln K_{\Omega}(z, z)=-2 \frac{\partial^{2}}{\partial z_{i} \partial \bar{z}_{j}} \ln \left(1-\rho_{\Omega}^{2}(z, w)\right)$.

Now, we can do the same for $S_{F, \Omega}$ instead of $K_{\Omega}$ and $H_{\Omega}$ instead of $L_{\Omega}$ and thus

$\frac{\partial^{2}}{\partial z_{i} \partial \bar{z}_{j}} \ln S_{F, \Omega}(z, z)=-2 \frac{\partial^{2}}{\partial z_{i} \partial \bar{z}_{j}} \ln \left(1-\left(\varrho_{\Omega}^{S_{F}}\right)^{2}(z, w)\right)$.

Using Lemma $19(b)$ one gets

$$
\begin{aligned}
\sum_{i, j=1}^{n} & \frac{\partial^{2}}{\partial z_{i} \partial \bar{z}_{j}} \ln H L_{\Omega}(z, w) \xi_{i} \bar{\xi}_{j} \\
= & 2 n \sum_{i, j=1}^{n} \frac{\partial^{2}}{\partial z_{i} \partial \bar{z}_{j}} \ln \left(1-\varrho_{\Omega}^{2}(z, w)\right) \xi_{i} \bar{\xi}_{j} \\
& \quad(2 n+2) \sum_{i, j=1}^{n} \frac{\partial^{2}}{\partial z_{i} \partial \bar{z}_{j}} \ln \left(1-\left(\varrho_{\Omega}^{S_{F}}\right)^{2}(z, w)\right) \xi_{i} \bar{\xi}_{j}
\end{aligned}
$$

or just

$\sum_{i, j=1}^{n} \frac{\partial^{2}}{\partial z_{i} \partial \bar{z}_{j}} \ln H L_{\Omega}(z, w) \xi_{i} \bar{\xi}_{j}=-n F_{B}^{2}(z ; \xi)+(n+1) F_{S_{F}}^{2}(z ; \xi)$.

But this right hand side expression is the quantity $E(z, \xi)$ introduced in [5]. Thus we get

$$
\begin{gathered}
\sum_{i, j=1}^{n} \frac{\partial^{2}}{\partial z_{i} \partial \bar{z}_{j}} \ln H L_{\Omega}(z, w) \xi_{i} \bar{\xi}_{j}=E(z, \xi) \\
=\sum_{i, j=1}^{n} \frac{\partial^{2}}{\partial z_{i} \partial \bar{z}_{j}} \ln S K_{\Omega}(z, z) \bar{\xi}_{i} \bar{\xi}_{j}
\end{gathered}
$$

Remark 28 In the case of the unit ball $\mathbb{B}^{n}$ in $\mathbb{C}^{n}, E(z, \xi) \equiv 0$, since $H L \equiv 1$ by the hypothesis of Lemma 19 . In particular, we have a direct connection between the Bergman and Szegó metrics on the unit ball in $\mathbb{C}^{n}$, namely: 
$F_{B}=\sqrt{\frac{n+1}{n}} F_{S_{F}}$.

This is also derived in [5]. Note that (4) clearly implies that $E(z ; \xi)$ defines a semi-positive definite form on a set $\left\{\xi \in T \bar{\Omega} ; F_{B}(z, \xi) \leq \sqrt{\frac{n+1}{n}} F_{S_{F}}(z, \xi)\right\}$. So for example if $\Omega$ is simply connected, and biholomorphic to the ball (see properties of $E(z ; \xi)$ in [5]).

\section{Closing remarks}

It has become increasingly clear that analysis on domains in $\mathbb{C}^{n}$ must be formulated in the language of invariant metrics. Thus it is worthwhile to develop and study new invariant metrics, and to compare them with the more familiar metrics that were developed in the twentieth century. This contribution is a step in that direction.

Acknowledgements We would like to thank the Referee for the valuable comments and suggestions regarding this paper.

\section{Declarations}

Conflict of interest The authors declare that they have no conflicts of interest.

Open Access This article is licensed under a Creative Commons Attribution 4.0 International License, which permits use, sharing, adaptation, distribution and reproduction in any medium or format, as long as you give appropriate credit to the original author(s) and the source, provide a link to the Creative Commons licence, and indicate if changes were made. The images or other third party material in this article are included in the article's Creative Commons licence, unless indicated otherwise in a credit line to the material. If material is not included in the article's Creative Commons licence and your intended use is not permitted by statutory regulation or exceeds the permitted use, you will need to obtain permission directly from the copyright holder. To view a copy of this licence, visit http://creativecommons.org/licenses/by/4.0/.

\section{References}

1. Skwarczyński, M.: Biholomorphic Invariants Related to the Bergman Function, Dissertationes Mathematicae (Rozprawy Matematyczne). Polish Scientific Publishing Company, Warsaw (1980)

2. Krantz, S.G.: Function Theory of Several Complex Variables, 2nd edn. AMS Chelsea Publishing, New York (1992)

3. Fefferman, C.: Parabolic invariant theory in complex analysis. Adv. Math. 31(2), 131-262 (1979)

4. Aronszajn, N.: Theory of reproducing kernels. Trans. Am. Math. Soc. 68, 337-404 (1950)

5. Barrett, D., Lee, L.: On the Szegő metric. J. Geom. Anal. 24(1), 104-117 (2014)

6. Krantz, S.G.: The Fefferman-Szegő metric and applications. Compl. Var. Elliptic Equ. 64, 965-978 (2019)

7. Jarnicki, M., Pflug, P.: Invariant distances and metrics in complex analysis. De Gruyter Exposit. Math. 9, 1-12 (2013)

8. Kobayashi, S.: Geometry of bounded domains. Trans. Am. Math. Soc. 92, 267-290 (1959)

9. Zeager, C.A.: The Azukawa Metric and the Pluricomplex Green Function. Thesis (Ph.D.)-University of Michigan. ProQuest LLC, Ann Arbor (2011)

10. Skwarczyński, M.: Metric completeness in holomorphic geometry. Bull. Soc. Sci. Lett. Łódź Rech. Deform. 51, 11-22 (2006)

11. Bedford, E., Fornæss, J.E.: A construction of peak functions on weakly pseudoconvex domains. Ann. Math. 107, 555-568 (1978)

12. Davie, A.M., Oksendal, B.K.: Peak interpolation sets for some algebras of analytic functions. Pac. J. Math. 41, 81-87 (1972)

13. Chen, B.-Y., Fu, S.: Comparison of the Bergman and Szegő kernels. Adv. Math. 228(4), 2366-2384 (2011)

14. Kerzman, N.: The Bergman kernel function. Differentiability at the boundary. Math. Ann. 195, 149-158 (1972)

15. de Monvel, L.B., Sjöstrand, J.: Sur la singularité des noyaux de Bergman et de Szegô. (French) Journées: Équations aux Dérivées Partielles de Rennes (1975), pp. 123-164. Asterisque, No. 34-35, Soc. Math. France, Paris (1976)

16. Mazur, T., Pflug, P., Skwarczyński, M.: Invariant distances related to the Bergman function. Proc. Am. Math. Soc. 94(1), 72-76 (1985)

17. Fefferman, C.: The Bergman kernel and biholomorphic mappings of pseudoconvex domains. Invent. Math. 26, 1-65 (1974)

Publisher's Note Springer Nature remains neutral with regard to jurisdictional claims in published maps and institutional affiliations. 